\title{
Comparison of OFDM and ideal SC-DFE achievable rates and performances without channel knowledge at the transmitter
}

\author{
Amanda S. de Paula and Cristiano M. Panazio
}

\begin{abstract}
This paper provides an achievable rate and performance analysis between OFDM and cyclic prefixed SC-DFE when no channel knowledge is available at the transmitter. Through some algebraic manipulation of the OFDM and SCDFE capacities and using the Jensen's inequality, we prove that the SC-DFE achievable rate is always superior to that of an OFDM scheme for 4- and 16-QAM for any given channel. For higher-order modulations, however, the advantage of the ideal SC-DFE is much smaller and also the results indicate that OFDM may surpass the ideal SC-DFE achievable rate by a small amount in some specific scenarios. Finally, we provide simulation results in order to assess, corroborate and illustrate the analytical results in terms of achievable rate and bit-error rate, where we conclude that a well designed OFDM system can be, in practical context, as effective as an ideal SC-DFE but with much smaller complexity when compared to a SC-DFE implementation with error-propagation mitigation.
\end{abstract}

Index Terms-Orthogonal frequency division multiplexing, cyclic prefixed single-carrier modulation, decision-feedback equalizer, achievable rate.

\section{INTRODUCTION}

$\mathbf{B}^{\mathrm{R}}$ Roadband single-carrier (SC) transmission scheme can naturally provide robustness against frequency selective channels since each transmitted symbol has a very short duration and it is spread all over the used bandwidth, as long as it is properly equalized [1]. On the other hand, orthogonal frequency division multiplexing (OFDM) without channel state information at the transmission sends symbols with equal mean power on an ensemble of narrowband orthogonal subcarriers, which some of them may suffer from deep fading, resulting in the data loss [2], [3], [4]. To overcome such problem, OFDM must solely rely on channel coding [2], [3], [5], [6], [7], so that the data lost on the attenuated subcarriers can be recovered from the good ones. Furthermore, the SC equalization presents a similar computational complexity when compared to OFDM, since it can also be performed in frequency-domain with the aid of the cyclic-prefix, such as used in OFDM, or with some small modifications when using the decision-feedback equalizer (DFE) [1], [8], which can improve SC performance with some additional complexity. Therefore, the immediate question that arises is how does

A. S. de Paula is with the Academic Unity of Cape of Saint Augustin (UACSA)-Rural Federal University of Pernambuco (UFRPE), Brazil (email: amanda.spaula@ufrpe.br)

C. M. Panazio is with Escola Politécnica of the University of São Paulo (EPUSP), Brazil (email:cpanazio@usp.br).

The authors would like to thank CAPES for scholarship funding.

Digital Object Identifier: 10.14209/jcis.2017.3
OFDM compare in terms of achievable rate and bit-error rate (BER) performance to the SC approach.

We focus our attention in this paper on the ideal SC-DFE scheme, which has no error propagation, since it can achieve the channel capacity under the constraint of a uniform power spectrum at the transmission and Gaussian signaling [7], [9]. In the case of M-ary quadrature amplitude modulation (QAM), this is no longer true and it cannot be said that it is the optimal receiver, despite it outperforms OFDM in the scenarios simulated in [1], [7], [10]. However, these papers also show an interesting trend in that the performance gap between such communications schemes becomes smaller and tends to vanish as the modulation order increases and/or the coding rates are kept low. Some numerical examples for specific channels and only for 16-QAM, but without providing any generalization for any channel through an analytical approach, are given in [10] to corroborate such conjecture. In [7], the cutoff rate [11], which is a less tight bound on the achievable rate, is used to analytically compare ideal SC-DFE and OFDM, but is limited for any three tap channel and just for QPSK modulation. Particularly, in comparison with the latter, we use the same approach to compare both systems, i.e., we employ the Jensen's inequality [12] analytical tool, but in place of the cutoff rate, we use Shannon's mutual information in this paper. This allows us to achieve a more rigorous and general conclusion applicable to the conjecture presented by [7], [10] is valid for any given channel but only for 4- and 16QAM schemes. For higher-order modulations, there are some particular cases where the OFDM scheme can present some marginal rate advantage over the ideal SC-DFE system.

It is worth to acknowledge and noting that this same problem has also been addressed in parallel to ours in [13], where, using Information-Estimation tools, they have established inequalities involving the OFDM and SC rates for several modulation schemes. However, our results, despite not being so extensive in terms of relative achievable rate differences, were generated by applying a similar, but simpler and more intuitive analysis method and also provides some more practical insights, showing through rate and BER simulations that when using the appropriate modulation and coding rate a practical OFDM scheme can achieve the same performance of an ideal SC-DFE that assumes unrealistic hypothesis. It is also worth noting that there are many BER comparisons of OFDM and SC-DFE in the literature, such as [1], [2], [6]. However, they fail to address how the modulation and coding scheme influence the power offset differences between OFDM and SC- 


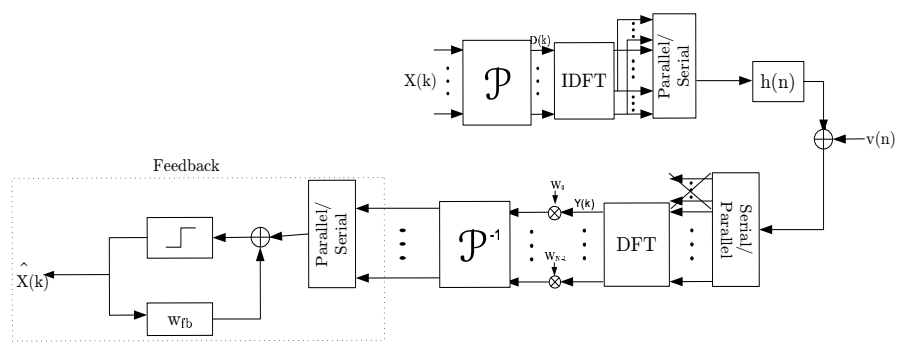

Fig. 1. Frequency-domain system model of OFDM and SC-DFE transceivers.

DFE and how we should set these parameters in order to make OFDM perform as good or better than the ideal SC-DFE.

This paper is organized as follows. In Section II, we describe the system model. In Section III, the comparison between the achievable rate of the considered schemes is done through the use of the channel mutual information for different modulation cardinalities and any channel, using concavity function analysis and Jensen's inequality. BER simulation results are shown in Section IV. Finally, conclusions are stated in Section IV.

\section{SYSTEM MODEL}

In order to describe both modulation schemes, we make use of a unified system model such as the one described by [14] and illustrated in Fig. 1.

The idea of such unified model is to use the OFDM modulation scheme with $N$ subcarriers as the basis for the SC scheme. The difference between the two systems lies on the linear precoding matrix $\mathcal{P}$ and its inverse $\mathcal{P}^{-1}$. For the OFDM, which does not originally have such matrix, $\mathcal{P}$ is simply the identity matrix. However, the SC scheme sends its symbols $\mathbf{x}$ without any subcarrier modulation and, thus, the matrix $\mathcal{P}$ must assume the form of a discrete Fourier transform (DFT), which we may represent by the matrix $\mathbf{F}^{1}$.

In such block transmission schemes the use of the $\mathrm{CP}$ approach has become an almost standard technique, since it allows the use of a very simple one-tap equalizer at the receiver. The $\mathrm{CP}$ consists in appending in the beginning of each transmitted block $\mathbf{s}=\left[s_{0}, \cdots, s_{N-1}\right]^{T}$ the last $N_{C P}$ samples of this same block. Then, the signal is linearly convoluted with a channel $\mathbf{h}=\left[h_{0}, \cdots, h_{L-1}, 0, \cdots, 0\right]^{T}$, with length $L<N_{C P}$, and a zero-mean circularly complex white Gaussian noise with variance $\sigma_{v}^{2}$ is added to form the received signal.

In the receiver, the $\mathrm{CP}$ is removed and we can then represent the received signal as

$$
\mathbf{r}=\mathcal{H} \mathbf{s}+\mathbf{v}
$$

where $\mathcal{H}$ is a circulant matrix whose first column is given by $\mathbf{h}$ and $\mathbf{v}=\left[v_{0}, \cdots, v_{N-1}\right]^{T}$ is the added noise.

${ }^{1}$ Other transforms of the family constant amplitude zero autocorrelation (CAZAC) or Walsh Hadamard can also be used as a precoding matrix, providing similar behavior as DFT-OFDM, i.e., SC approach. However, their linear minimum mean square error (MMSE) and zero-forcing (ZF) demodulation schemes will result in inferior performance when compared to the ideal SC-DFE, since they are analogous to the SC linear equalizer scheme [7].
In the frequency domain, after the DFT, the received signal can be represented by

$$
\mathbf{y}=\mathbf{H x}+\mathbf{F v},
$$

where, due to the use of the inverse discrete Fourier transform (IDFT) at the transmitter, $\mathbf{H}=\mathbf{F} \mathcal{H} \mathbf{F}^{H}=\operatorname{diag}\{\mathbf{F h}\}$ is the diagonalized version of $\mathcal{H}$, and $\mathbf{F v}$ is also a zero-mean circularly complex white Gaussian noise with variance $\sigma_{v}^{2}$, due to the use of the unitary DFT.

Since the channel can be modeled as a diagonal matrix in the frequency domain, i.e., the subcarriers are orthogonal, the equalizer, represented by the coefficients $W_{k}$ used to estimate the transmitted signal in each frequency bin can be reduced to phase and magnitude compensations, the so-called one-tap equalizer. Here, it is worth noting a distinction between the OFDM and SC systems. In the OFDM, such equalization does not change the signal-to-noise ratio (SNR) in each subcarrier and, hence, there is no performance penalty for the signal decoding process. Then, in order to simplify the calculations, we can assume a zero-forcing (ZF) criterium.

Contrarily, for the SC scheme, such ZF criterium would imply in large amounts of noise amplification and a huge decrease in the performance. Thus, it is usually used the minimum mean square error (MMSE) criterium, which takes into account such noise amplification.

Even though, the performance of such SC system with this linear equalizer falls short in comparison to the OFDM, particularly when higher order modulations are used [7]. In this sense, we use an ideal and unbiased MMSE SC-DFE with coefficients calculated in [15], [16]. The feedback filter is shown in the dotted box at the receiver in Fig. 1. This equalizer allows us to achieve channel capacity under the constraint of uniform power spectrum for Gaussian signaling [7], [9].

\section{ACHIEVABLE RATE COMPARISON BETWEEN THE IDEAL SC-DFE AND OFDM USING QAM}

Let us consider a squared $M$-ary QAM scheme and an additive white Gaussian noise (AWGN) channel. The associated maximum rate, i.e., the mutual information, is two times the maximum rate of a $\sqrt{M}$-pulse amplitude modulation [12]:

$$
\begin{gathered}
R^{\mathrm{M}-\mathrm{QAM}}(\gamma)=-2 \int_{-\infty}^{+\infty} f_{R}(r, \gamma) \log _{2} f_{R}(r, \gamma) d r \\
-\log _{2} \frac{2 \pi e \sigma_{x}^{2}}{\gamma}
\end{gathered}
$$

where $\gamma=\frac{\sigma_{x}^{2}}{\sigma_{v}^{2}}$ is the $\operatorname{SNR}$ and $f_{R}(r, \gamma)=$ $\sqrt{\frac{\gamma}{2 M \pi \sigma_{x}^{2}}} \sum_{m=-\sqrt{M} / 2}^{\sqrt{M} / 2-1} e^{-\frac{\gamma(r-(2 m+1))^{2}}{2 \sigma_{x}^{2}}}$ is a probability density function, which corresponds to the sum of Gaussian distributions with variance $\sigma_{v}^{2}=\gamma / \sigma_{x}^{2}$, centered at the $\sqrt{M}$-PAM points and pondered by $1 / \sqrt{M}$ in order to have unitary area.

Then, in order to calculate the OFDM achievable rate, let us define the SNR for each subcarrier as $\gamma_{k}=\gamma\left|H_{k}\right|^{2}$, where $H_{k}$ is the $k^{t h}$ element of the diagonal of $\mathbf{H}$. Note that since 
we are considering $\sum_{k=0}^{N-1}\left|H_{k}\right|^{2}=1, \gamma$ is the average SNR of the system.

Since all $N$ subcarriers transmit the same $M$-ary QAM scheme, the OFDM achievable rate is the average of the rate in the subcarriers:

$$
R_{\mathrm{OFDM}}^{\mathrm{M}-\mathrm{QAM}}=\frac{1}{N} \sum_{k=0}^{N-1} R^{\mathrm{M}-\mathrm{QAM}}\left(\gamma_{k}\right) .
$$

It is worth noting that a single channel code can be used to code the information bits to form $\mathbf{x}$ as long as the information rate is lower than (4) [17].

Now, for the ideal SC-DFE, let us consider that any residual intersymbol interference in the ideal SC-DFE output can be modeled as a Gaussian noise due to the central limit theorem. It is worth noting that such hypothesis seems reasonable since the SC-DFE feedforward filter uses a large number of coefficients in our context. Then, the achievable rate of the ideal SC-DFE system can be evaluated by applying the SNR of an ideal DFE [7]:

$$
\gamma_{\mathrm{DFE}}=\exp \left\{\frac{1}{N} \sum_{k=0}^{N-1} \log \left(1+\gamma_{k}\right)\right\}-1,
$$

in (3), so that we have:

$$
R_{\mathrm{DFE}}^{\mathrm{M}-\mathrm{QAM}}=R^{\mathrm{M}-\mathrm{QAM}}\left(\gamma_{\mathrm{DFE}}\right) .
$$

As noted in [7], the subcarriers with the best SNRs that achieve a rate near the saturation of (3), i.e., when (3) is near $\log _{2} M$ bits/s/Hz, cannot compensate for the rate loss of the attenuated subcarriers and hence (4) will be smaller than (6). To the best of the authors knowledge, such limitation has been only observed in [10] and [18], and, in parallel, without knowledge of these previous publications, by the authors in [7]. The problem is that [18] misses a detailed explanation and, in [7], [10], although they provide compelling evidence, they do not provide conclusive proofs on the achievable rate comparison.

One way to overcome this achievable rate limitation of OFDM is to use a larger cardinality, i.e., a larger $M$ for the same average SNR value so that the best subcarriers can provide a higher rate to compensate the attenuated ones (cf., [7], [10]). However, the question that arises is how the OFDM scheme compares to the ideal SC-DFE. To answer this question, firstly, let us define $\phi(x)=\log (x+1)$ and its inverse $\phi^{-1}(x)=\exp (x)-1$, thus, $\gamma_{D F E}$ can be expressed as

$$
\gamma_{D F E}=\phi^{-1}\left(\frac{1}{N} \sum_{k=0}^{N-1} \phi\left(\gamma_{k}\right)\right) .
$$

In the following, we define:

$$
\tau(x)=R^{\mathrm{M}-\mathrm{QAM}}\left(\phi^{-1}(x)\right),
$$

so that (6) and (4) can be rewritten as

$$
R_{\mathrm{DFE}}^{\mathrm{M}-\mathrm{QAM}}(\gamma)=\tau\left(\frac{1}{N} \sum_{k=0}^{N-1} \phi\left(\gamma_{k}\right)\right)
$$

and

$$
R_{\mathrm{OFDM}}^{\mathrm{M}-\mathrm{QAM}}(\gamma)=\frac{1}{N} \sum_{k=0}^{N-1} \tau\left(\phi\left(\gamma_{k}\right)\right) .
$$

In order to state the relation between (9) and (10), it is interesting to analyze the concavity properties of the function $\tau(x)$, which can be rewritten as:

$$
\tau(x)=R^{\mathrm{M}-\mathrm{QAM}}(\exp (x)-1) .
$$

This can be accomplished through the analysis of its second derivative. If it is positive, we can state that the function $\tau(x)$ is convex in the considered interval. Otherwise, we state that $\tau(x)$ is concave. In this case, using Jensen's inequality, we state that:

$$
\tau\left(\frac{1}{N} \sum_{k=0}^{N-1} \phi\left(\gamma_{k}\right)\right) \geq \frac{1}{N} \sum_{k=0}^{N-1} \tau\left(\phi\left(\gamma_{k}\right)\right),
$$

which means that $R_{\mathrm{DFE}}^{\mathrm{M}-\mathrm{QAM}}(\gamma) \geq R_{\mathrm{OFDM}}^{\mathrm{M}-\mathrm{QAM}}(\gamma)$.

In Fig. 2, we show the second derivative of $\tau(x)$ numerically evaluated for different QAM cardinalities. We can observe that it is a non-positive function for 4 and 16-QAM. In particular, for 64-QAM, the second derivative is positive only for a small interval of $x$ comprised between 2.57 and 2.72 , where it attains a maximum value of $3.58 \times 10^{-4}$, so that it renders very unlikely to see some rate advantage of OFDM in such a case. For higher cardinalities, there are larger intervals of $x$ that the second derivative achieves higher positive values allowing the OFDM scheme to present a higher rate than the ideal SC-DFE depending on the channel. Anyway, the second derivative still assumes only small positive values from which we conclude that the performance advantage tends to be small. For instance, by using the 1024-QAM and the most favorable channel to OFDM, as described in [13], we achieved a maximum rate advantage of $1.22 \%$. It is important to note that for such high-order modulations, the achievable rates of both schemes are practically the same in a broad range of SNRs. The only real advantage of the ideal DFE occurs when the SNR is high enough so that many subcarriers of the OFDM are getting closer to the maximum rate saturation of the selected modulation, but then the use of a higher modulation cardinality would overcome such problem. Note also, for a given channel and SNR, using a higher-order modulation will never provide a smaller rate than a lower-order modulation for any transmission scheme.

In order to illustrate these results, we have calculated through simulations the ergodic rate of each scheme for a 128 coefficients channel, obtained using a uniform power profile and Rayleigh distribution, and $N=512$ for the 4-QAM to the 1024-QAM schemes. The results are presented in Fig. 3 as it shows the ratio between the ergodic rates. As expected, only from 256-QAM the OFDM scheme presents some rate advantage on a certain range of SNRs. On the other hand, the DFE advantage is not considerably large, with a maximum of $15.5 \%$ for 4-QAM, but topping at 5\% for 1024-QAM for high SNR regimes. Therefore, in practice, OFDM can have almost the same or higher rate than an ideal DFE when using large modulation cardinalities and low coding rates, whereas it is 


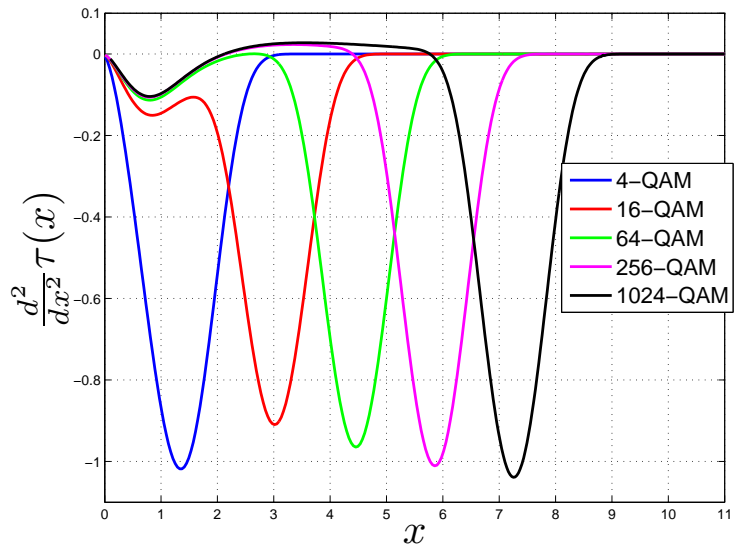

Fig. 2. Second derivative of $\tau(x)$.

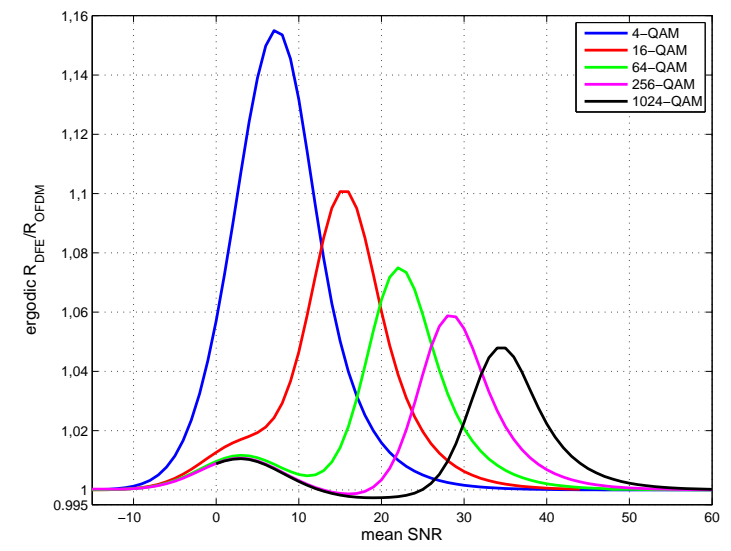

Fig. 3. Achievable rate ratio between ergodic $R_{\mathrm{DFE}}$ and $R_{\mathrm{OFDM}}$ for various modulation cardinalities and a 128 taps uniform power profile Rayleigh channel and $\mathrm{N}=512$.

quite complex to come close to the ideal DFE performance due to the need to eliminate error propagation. It is also important to note that these performance numbers differences depend on the channel length and power profile, but the general behavior, i.e., the need to use larger modulation orders and lower coding rates with OFDM to achieve about the same ideal DFE performance, is consistent for any channel selective channel as will be illustrated in the next section.

Despite the more general results obtained above by using the mutual information, they cannot be immediately converted in relative BER $\times$ SNR (or similarly, BER x $E_{b} / N_{o}$ ) performances, which are more intuitive and of more practical interest. Hence, in order to illustrate and corroborate the theoretical results obtained in this section and show how the achievable rate results translate into such merit figures, we provide additional simulations in the next section.

\section{Bit-error Rate Simulation Results}

Being the BER the ultimate result of a communication system, in this section, we show that the achievable rate comparison results, despite some hypothesis and approximations (infinite length codes, Gaussian ISI at the DFE output for QAM), can explain such BER differences in more practical coded systems and also with a block-fading channel. For all simulations, we have used $N=512$ subcarriers in the OFDM system, as well as considered $N=512$ symbols in the SC-DFE block. We use a bit-interleaved coded modulation (BICM) approach [19]. A random interleaver was used for both schemes and its length comprises the bits necessary to generate $N$ symbols. For each transmitted block, the interleaver configuration was randomly chosen. We also assume perfect channel estimation. All static channels have been normalized to have unitary norm and the block-fading channel has unitary average power.

First, we use 16-QAM and a channel with complex coefficients, which zeros are at $\exp (j 0.5533 \pi)$ and $\exp (j 0.1133 \pi)$. This channel has its zeros over the unitary circle generating deep fades and large gains that greatly scatter $\left|H_{k}\right|^{2}$ values, creating large performance differences between the OFDM and SC-DFE schemes for mild to high average SNR values. We then use two convolutional coding schemes: a rate $1 / 2$ with polynomial $(133,171)$ and a rate $1 / 4$ with polynomial $(117,127,155,171)[20$, pp. 539-540]. The former code can only provide low BER for higher values of average SNR, in which certain OFDM subcarriers will operate closer to or in the saturation region of (4), and the latter makes both schemes have similar performances for lower average SNRs, since (6) and (4) are approximately the same. The results are depicted in Fig. 4. It is worth noting that OFDM slightly surpasses the SC-DFE performance for low average SNR regimes (lower than $6 \mathrm{~dB}$ ) with the rate $1 / 4$ code. This may be explained by two reasons. The first reason is that for low SNR regimes, the achievable rate difference between OFDM and SC-DFE is almost negligible for this channel. The second reason is that BICM is not the optimal coding scheme for SC-DFE in this context, since the equalizer output seems as an additive white Gaussian noise (AWGN) channel for the decoder and it is known that BICM has a performance loss in such a case [19]. On the other hand, the BICM decoder sees the dispersion of $\left|H_{k}\right|^{2}$ for each subcarrier as a time-varying channel and can make the best use of the BICM approach. In contrast, for the rate $1 / 2$ code, when the BER starts to decrease from 0.5 , which occurs around a SNR equal to $6 \mathrm{~dB}$, the achievable rate difference between the OFDM and SC-DFE schemes becomes noticeable, and the SC-DFE BER is smaller than the OFDM BER. This behavior can also be observed for the rate $1 / 4$ code, where the OFDM performance starts to degrade in comparison to the SC-DFE performance, which corroborates the behavior expected by the achievable rate analysis.

In the following, we use 4-QAM and the convolutional code $(13,17)$ [20, p. 540] for two different channels. The first channel is the channel with zeros at $0.5 \exp ( \pm j 0.5 \pi)$. The second one presents two complex conjugated zeros in the unitary circle: $\exp ( \pm j 0.22 \pi)$. The first channel produces a small dispersion of $\left|H_{k}\right|^{2}$ and the achievable rate calculation for both schemes results in almost the same values for a large range of SNRs. The second channel generates large dispersions of $\left|H_{k}\right|^{2}$ and leads to larger performance differences. The results are shown in Fig. 5. We may observe that the less frequency selective channel results in equivalent performances, except that OFDM starts to show a performance loss for a 


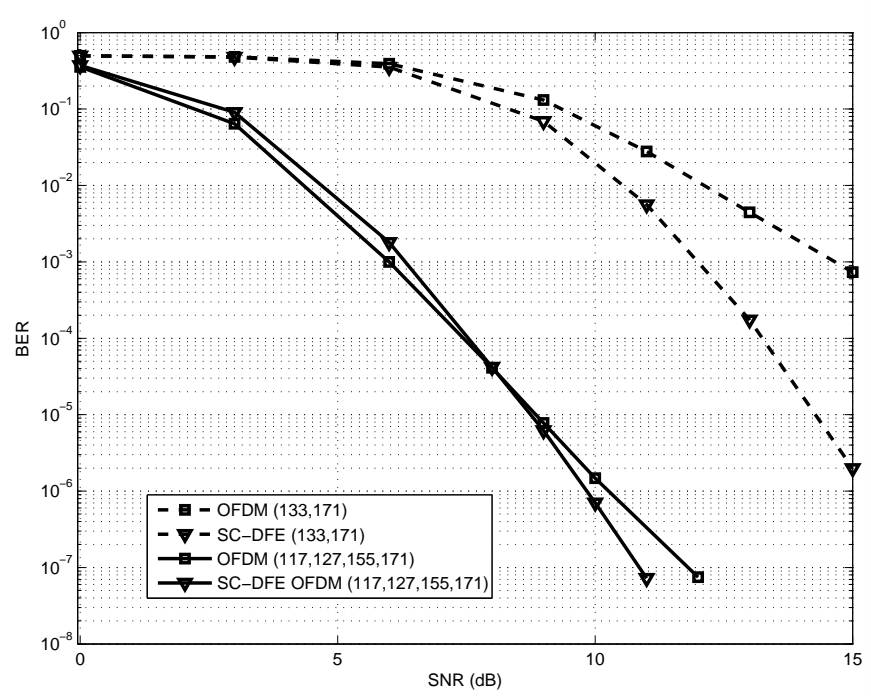

Fig. 4. BER performance versus average SNR for 16-QAM in which the solid lines $(-)$ are for the convolutional code with polynomial $(117,127,155,171)$ and the dashed lines (--) are for the convolutional code with polynomial $(133,171)$

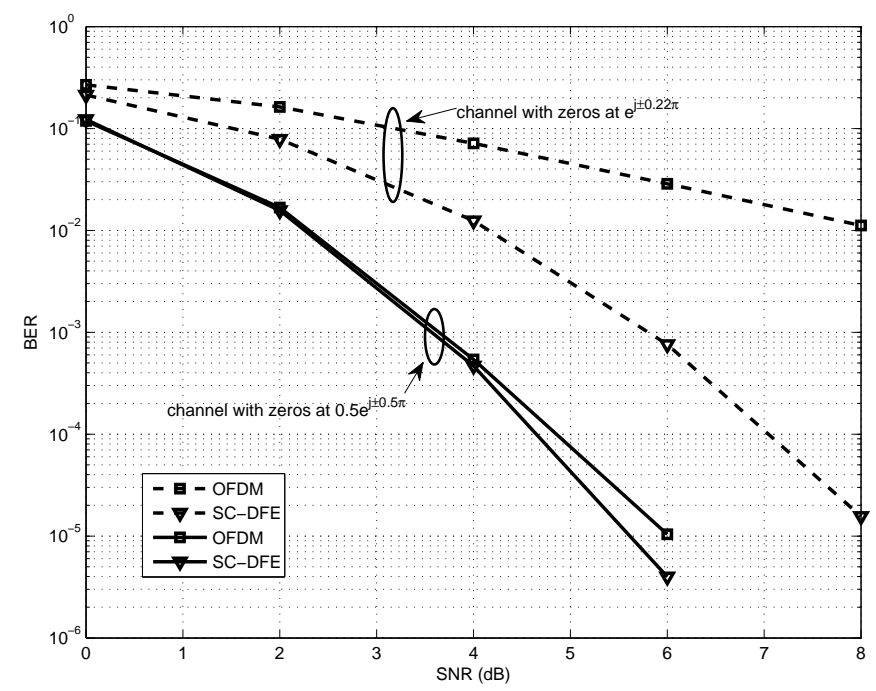

Fig. 5. BER performance versus average SNR (or $E_{b} / N_{o}$ ) for 4-QAM in which the solid lines $(-)$ are for the channel with zeros at $0.5 \exp ( \pm j 0.5 \pi)$ and the dashed lines (--) are for the channel with zeros at $\exp ( \pm j 0.22 \pi)$. The convolutional code for both channels has the polynomial $(13,17)$

SNR higher than $4 \mathrm{~dB}$, but with an already low BER. For the second channel, the ideal SC-DFE is much better than the OFDM scheme that provides unacceptable performance, since this channel provides a large deviation of $\left|H_{k}\right|^{2}$.

Finally, in Fig. 6, we show the BER for a more practical scenario, i.e., a block-fading channel with three equal power complex Gaussian taps for a constant spectral efficiency equal to $3 \mathrm{bits} / \mathrm{s} / \mathrm{Hz}$. Such efficiency is achieved with two different configurations. The first one is 16-QAM with a punctured code $(133,171)$ to achieve a rate equal to $3 / 4$. The second one is obtained with 64-QAM and the code $(133,171)$ without puncturing. We also present the performance of the non ideal SC-DFE where the symbol decisions are fedback and thus it is subject to error propagation. For the 16-QAM scheme,

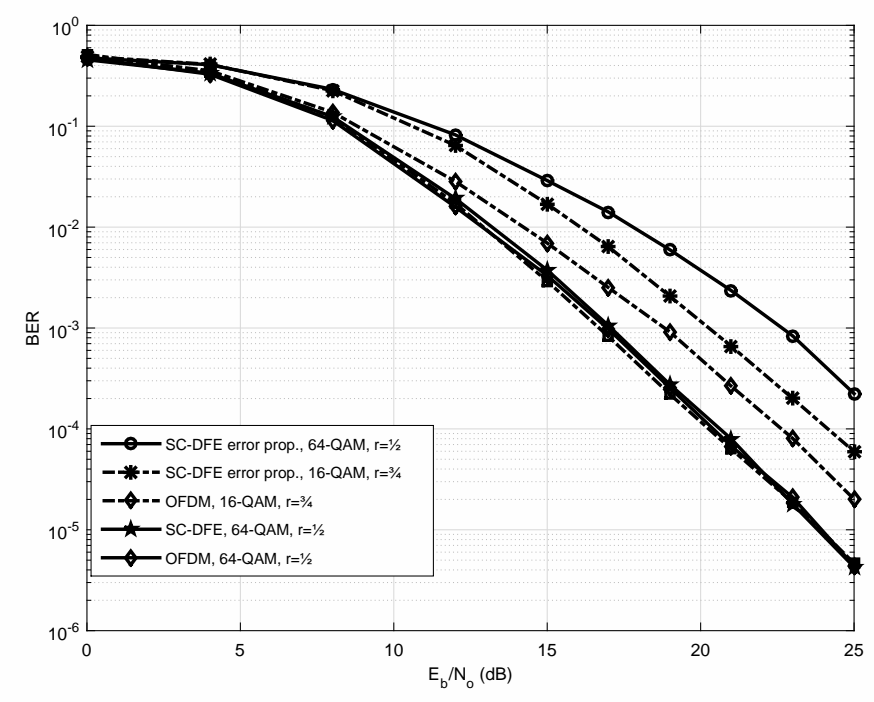

Fig. 6. BER performance versus average $E_{b} / N_{o}$ spectral efficiency equal to $3 \mathrm{bit} / \mathrm{s} / \mathrm{Hz}$ and block-fading channel with three equal power taps in which the dashed lines (--) are for the 16-QAM with coding rate 3/4 obtained by puncturing the code $(133,171)$ and the solid lines $(-)$ are for the 64-QAM with coding $(133,171)$.

the OFDM scheme is not as good as the ideal SC-DFE version being more than $2 \mathrm{~dB}$ worse at a BER equal to $10^{-3}$. Then, the 64-QAM scheme and the code with rate $1 / 2$ allow both OFDM and the ideal SC-DFE to achieve equal BER performances, practically matching the ideal SC-DFE 16-QAM with code rate $3 / 4$. Note also that both versions of the non ideal SC-DFE that present error propagation are outperformed by both OFDM implementations ${ }^{2}$, since the error bursts tend to overcome the coding correction capabilities even with the use of an interleaver. This result indicates that a practical OFDM when using high-order modulation and lower coding rates for a given target $\mathrm{SNR}$ or $\mathrm{E}_{\mathrm{b}} / \mathrm{N}_{0}$ in a frequencyselective channel can provide the performance of an ideal, but unrealistic SC-DFE.

\section{CONCLUSION}

In this paper, we have compared the channel capacities of the ideal SC-DFE and OFDM schemes for square $M$ QAM modulations in frequency selective channels. By using Jensen's inequality, we were able to prove that the ideal SCDFE achievable rate is larger than the OFDM achievable rate for 4- and 16-QAM for any given frequency-selective channel. For higher cardinalities, OFDM provides an achievable rate closer to or slightly higher than the ideal SC-DFE, since highorder modulations will offer at least the same rate than lower cardinalities for any transmission scheme for a given SNR and channel. Then, from the results in this paper and previous ones, optimal OFDM implementations should use larger modulation cardinalities with the appropriate coding rate and large coding gains to stay within the system achievable rate. Using such strategy, we have shown through BER simulations that both

\footnotetext{
${ }^{2}$ Note that the error propagation gives only a power offset penalty, since both versions of the non ideal SC-DFE can achieve the same diversity of the ideal SC-DFE and OFDM schemes at high $\mathrm{E}_{\mathrm{b}} / \mathrm{N}_{0}$ values.
} 
system performances are about the same. Nonetheless, it is worth noting that the ideal SC-DFE is not realizable and the SC-DFE scheme suffers a large performance hit due to error propagation, specially in channels with deep fades that usually result in large feedback coefficients. This error propagation can be mitigated by using turbo-like iterative techniques, but at the expense of additional computational complexity and higher latency. In this sense, OFDM seems to be a more reasonable solution, as long as the system parameter, i.e., modulation and coding scheme are wisely chosen and when the peakto-average power ratio is not a system limitation.

\section{REFERENCES}

[1] D. Falconer, S. L. Ariyavisitakul, A. Benjamin-Seeyar, and B. Edison, "Frequency domain equalization for single-carrier broadband wireless systems," IEEE Commun. Mag., vol. 40, pp. 58-66, Apr. 2002, doi:10.1109/35.995852.

[2] H. Sari, G. Karam, and I. Jeanclaude, "Transmission techniques for digital terrestrial TV broadcasting," IEEE Commun. Mag., vol. 33, no. 2, pp. 100-109, Feb. 1995, doi:10.1109/35.350382.

[3] Z. Wang, X. Ma, and G. Giannakis, "OFDM or single-carrier block transmissions?" IEEE Trans. Commun., vol. 52, no. 3, pp. 380-394, Mar. 2004, doi:10.1109/TCOMM.2004.823586.

[4] B. Devillers and L. Vandendorpe, "Bit rate comparison of adaptive OFDM and cyclic prefixed single-carrier with DFE," Сотmunications Letters, IEEE, vol. 13, no. 11, pp. 838-840, Nov. 2009, doi:10.1109/LCOMM.2009.091008.

[5] V. Aue, G. P. Fettweis, and R. Valenzuela, "A comparison of the performance of linearly equalized single carrier and coded OFDM over frequency selective fading channels using the random coding technique," in Proc. Inter. Conf. on Commun. (ICC), vol. 2, June 1998, pp. 753-757, doi:10.1109/ICC.1998.685111.

[6] J. Tubbax, L. V. der Perre, M. Engels, H. De Man, and M. Moonen, "OFDM versus single carrier: A realistic multi-antenna comparison," EURASIP Journal on Applied Signal Processing, vol. 2004, no. 9, pp. 1275-1287, 2004, doi:10.1155/S1110865704402315.

[7] A. de Paula and C. Panazio, "A comparison between OFDM and singlecarrier with cyclic prefix using channel coding and frequency-selective block fading channels," Journal of Comm. and Information Systems, vol. 1, April 2011, doi:10.14209/jcis.2011.3.

[8] H. Witschnig, T. Mayer, A. Springer, A. Koppler, L. Maurer, M. Huemer, and R. Weigel, "A different look on cyclic prefix for SC/FDE," in Proc. The 13th IEEE Inter. Symp. on Personal, Indoor and Mobile Radio Commun. (PIMRC)., vol. 2, Lisbon, Portugal, Sep. 2002, pp. 824-828, doi:10.1109/PIMRC.2002.1047337.

[9] S. Shamai and R. Laroia, "The intersymbol interference channel: lower bounds on capacity and channel precoding loss," Information Theory, IEEE Transactions on, vol. 42, no. 5, pp. 1388-1404, Sep. 1996, doi:10.1109/18.532881.

[10] M. Franceschini, R. Pighi, G. Ferrari, and R. Raheli, "On information theoretic aspects of single- and multi-carrier communications," in Information Theory and Applications Workshop, 2008, San Diego, USA Feb. 2008, pp. 94-99, doi:10.1109/ITA.2008.4601030.

[11] J. Wozencraft and I. M. Jacobs, Principles of Communication Engineering. Wiley, 1965.

[12] T. M. Cover and J. A. Thomas, Elements of Information Theory, 2nd ed. Wiley, 2006

[13] Y. Carmon, S. Shamai, and T. Weissman, "Comparison of the achievable rates in ofdm and single carrier modulation with i.i.d. inputs," Information Theory, IEEE Transactions on, vol. 61, no. 4, pp. 1795-1818, Apr. 2015, doi:10.1109/TIT.2015.2403354.

[14] Y.-P. Lin and S.-M. Phoong, "BER minimized OFDM systems with channel independent precoders," IEEE Trans. Signal Process., vol. 51, no. 9, pp. 2369-2380, Sep. 2003, doi:10.1109/TSP.2003.815391.

[15] N. Benvenuto and S. Tomasin, "On the comparison between OFDM and single carrier modulation with a DFE using a frequency-domain feedforward filter," IEEE Trans. Commun., vol. 50, no. 6, pp. 947-955, Jun. 2002, doi:10.1109/TCOMM.2002.1010614.

[16] D. Falconer and S. Ariyavisitakul, "Broadband wireless using single carrier and frequency domain equalization," in The 5th Inter. Symp. on Wireless Personal Multimedia Commun., vol. 1, Honolulu, USA, Oct. 2002, pp. 27 - 36, doi:10.1109/WPMC.2002.1088127.
[17] W. L. Root and P. P. Varaiya, "Capacity of classes of Gaussian channels," SIAM J. Appl. Math., vol. 16, no. 6, pp. 1350-1393, Nov. 1968, doi:10.1137/0116114.

[18] R. Wesel and J. Cioffi, "Fundamentals of coding for broadcast OFDM," in Conference Record of the Twenty-Ninth Asilomar Conference on Signals, Systems and Computers, vol. 1, Oct. 1995, pp. 2-6 vol.1, doi:10.1109/ACSSC.1995.540504.

[19] G. Caire, G. Taricco, and E. Biglieri, "Bit-interleaved coded modulation," IEEE Trans. Inf. Theory, vol. 44, no. 3, pp. 927-946, May 1998 doi:10.1109/18.669123.

[20] S. Lin and D. J. C. Jr., Error Control Coding, 2nd ed. Prentice Hall, 2004.

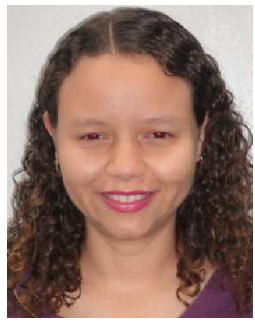

Amanda de Paula received the B.Sc. degree from Federal University of Pernambuco (UFPE) in 2008 and the M.Sc. degree and Ph.D. from Escola Politécnica of the University of São Paulo (EPUSP) in 2010 and 2014, respectively. In 2014, she has become Assistant Professor in Universidade Federal Rural de Pernambuco (UFRPE). Her research interests include multicarrier modulations, cognitive radio, detection and estimation theory.

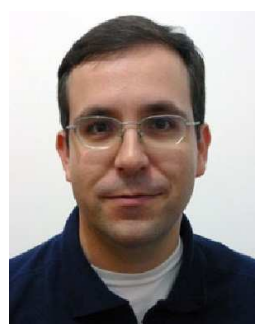

Cristiano Panazio received a B.Sc. and an M.Sc degree in Electrical Engineering from the State University of Campinas (UNICAMP), Brazil, in 1999 and 2001, respectively. He received his $\mathrm{Ph} . \mathrm{D}$. in 2005, also in Electrical Engineering, from the Conservatoire National des Arts Métiers (CNAM), Paris, France. In 2006, he became assistant professor at Escola Politécnica of the University of São Paulo. His research interests include equalization, multicarrier modulations, synchronization techniques, and cognitive radio. He is a member of the Brazilian Telecommunications Society (SBrT) since 1999 and where he currently serves as Vice-President of Development and Diffusion. He is also Associate Editor of IEEE Transactions on Circuits and Systems I. 